\title{
Infrared Dynamics in Vector-Like Gauge Theories: QCD and Beyond
}

\author{
Victor Elias \\ Department of Applied Mathematics, The University of Western Ontario \\ London, Ontario N6A 5B7 Canada
}

\begin{abstract}
Padé-approximant methods are used to extract information about leading positive zeros or poles of QCD and SQCD $\beta$-functions from the known terms of their perturbative series. For QCD, such methods are seen to corroborate the flavour-threshold behaviour obtained via lattice approaches for the occurrence of infrared-stable fixed points. All possible Padé-approximant versions of the known (one- to four-loop) terms of the QCD $\overline{M S}$ $\beta$-function series are consistent with this threshold occurring at or above $n_{f}=6$. This conclusion continues to be true even if higher-degree Padé-approximants are introduced to accommodate an arbitrary five-loop contribution to the QCD $\beta$-function for a given number of flavours.
\end{abstract}

The dynamics characterising the infrared region of asymptotically-free gauge theories are becoming an area of active investigation. Historically speaking, the property of asymptotic freedom has been linked with "infrared slavery" at large distances - the non-observability of gauge-group nonsinglet particles as asymptotic free-particle states because of the growth of the gauge coupling constant to arbitrarily large values at some infrared momentum scale. Such methodological simplification, however, rests upon the existence of a Landau singularity in the evolution of the gauge couplant, a singularity that is likely an artefact of truncating the gauge couplant's $\beta$-function to any given order. Consider, for example, the QCD $\beta$-function to two-loop order for three active flavours:

$$
\mu^{2} \frac{d x}{d \mu^{2}}=-\frac{9}{4} x^{2}-4 x^{3}, \quad x \equiv \alpha_{s}(\mu) / \pi .
$$

Given some empirical initial value, e.g. $x_{\tau}=\alpha_{s}\left(m_{\tau}\right) / \pi$, eq.(1) has the analytic solution

$$
\log \left(\mu^{2} / m_{\tau}^{2}\right)=\frac{4}{9}\left[\frac{1}{x(\mu)}-\frac{1}{x_{\tau}}\right]+\frac{64}{81} \log \left[\frac{x(\mu)\left(x_{\tau}+9 / 16\right)}{x_{\tau}(x(\mu)+9 / 16)}\right] .
$$

As $\mu$ decreases from $m_{\tau}$ in this expression, the couplant $x(\mu)$ grows from $x_{\tau}$ to become infinite at

$$
\mu_{L}=m_{\tau}\left(1+9 /\left(16 x_{\tau}\right)\right)^{32 / 81} \exp \left[-2 /\left(9 x_{\tau}\right)\right]
$$

the Landau singularity. The temptation is to identify $\mu_{L}$ with the infrared boundary of QCD as a perturbative gauge theory of quarks and gluons. Note that if $\alpha_{s}\left(m_{\tau}\right)=\pi x_{\tau}=0.35$, the central PDG value [1], then $\mu_{L}$ is found from (3) to be $493 \mathrm{MeV}$.

However, this Landau singularity is really a consequence of truncating the perturbative $\beta$-function series (1) to its renormalization scheme independent two-loop order terms. Subsequent $\beta$-function terms are renormalizationscheme-dependent and presumably negotiable (I will have more to say on this later on). For example, we can conjecture two toy-model $\beta$-functions

$$
\begin{gathered}
\mu^{2} \frac{d x}{d \mu^{2}}=-\frac{9}{4} \frac{x^{2}}{(1-16 x / 9)} \\
\mu^{2} \frac{d x}{d \mu^{2}}=-\frac{9}{4} x^{2}\left(1+\frac{16}{9} x+R_{2} x^{2}\right), \quad R_{2}<0
\end{gathered}
$$

which both agree to two-loop order with eq. (2), but have infrared behaviour manifestly different from the Landau pole characterising (2). The former equation has a $\beta$-function pole at $x=9 / 16=0.5625$. Consequently, as $\mu$ decreases from $m_{\tau}, x$ increases from $x_{\tau}$ to a maximum value of 0.5625 , which is achieved at a critical infrared momentum scale

$$
\mu_{c}=m_{\tau}\left(\frac{9}{16 x_{\tau}}\right)^{32 / 81} \exp \left[\frac{32}{81}-\frac{2}{9 x_{\tau}}\right]
$$


The domain of $x(\mu)$ is restricted to $\mu>\mu_{c}$, in which case $\mu_{c}$ represents an infrared-boundary of QCD at which the gauge couplant achieves its largest possible but finite value. The existence of an infrared boundary is no longer coupled with the arbitrary growth of the interaction coupling.

By contrast, the $\beta$-function of eq. (5) has a zero at $x_{I R F P}=-8 /\left(9 R_{2}\right)+\sqrt{64 /\left(81 R_{2}^{2}\right)-1 / R_{2}}\left(R_{2}\right.$ is negative), meaning that $x(\mu)$ increases to eventually level off at $x_{I R F P}$ as $\mu \rightarrow 0$. For this case, which corresponds to the existence of an infrared-stable fixed point (IRFP), there is no infrared boundary to gauge-theoretical QCD - the domain of $x(\mu)$ includes all (positive) values of $\mu$.

A point that needs to be made here is that if (1), (4), and (5) were to represent all-orders $\beta$-functions arising from legitimate but differing renormalization schemes, 1 then the infrared behaviour of the couplant would itself be scheme-dependent. Indeed, one could easily construct a $\beta$-function candidate consistent with (1) to two-loop order whose higher order terms are sufficiently large and negative [e.g. an arbitrarily large negative $R_{2}$ in (5)] to ensure that an IRFP occurs at an arbitrarily small value for $x$, suggesting that QCD in such a scheme would remain a tractable perturbative gauge theory at arbitrarily large distances. Since the infrared behaviour of QCD is empirically known to be confining at sufficiently large distances, such apparent scheme-dependence must be overstated. We therefore take the point of view here that differing but self-consistently realized renormalization schemes will necessarily lead to the same physical results - in particular, to equivalent infrared dynamics.

A vector-like gauge theory that illustrates all this is that of $N=1$ supersymmetric SU(3) Yang-Mills theory, supersymmetric gluodynamics characterised only by gluon and gluino fields. Because of its supersymmetry, the all-orders $\beta$-function for this theory can be obtained algebraically by requiring that the anomaly multiplet within the theory respect the Adler-Bardeen theorem [3,4], or alternatively via instanton-calculus considerations [5]. Surprisingly, the same $\beta$-function arises from either approach, and even more surprisingly, this $\beta$-function, like (4), is characterised by a pole:

$$
\mu^{2} \frac{d x}{d \mu^{2}}=-\frac{9 x^{2}}{1-6 x}, x \equiv g^{2}(\mu) / 16 \pi^{2}
$$

As noted in [6], such a pole necessarily implies a critical momentum scale $\mu_{c}$ for the asymptotically free phase of the theory that would constitute an infrared boundary to the region for which the theory is perturbative. The pole value of the couplant $\left[x\left(\mu_{c}\right)=1 / 6\right]$ constitutes an infrared-attractive point terminating the evolution of the (real) gauge coupling constant in its asymptotically-free phase. We henceforth denote (7) as the NSVZ $\beta$-function, after the authors of ref. 5 .

The $\beta$-function for supersymmetric gluodynamics has also been calculated to three non-leading orders within the dimensional-reduction (DRED) renormalization scheme [7]:

$$
\mu^{2} \frac{d x}{d \mu^{2}}=-9 x^{2}\left[1+6 x+63 x^{2}+918 x^{3}+\ldots\right]
$$

The higher order terms of the $\beta$-function (8) differ from those of the geometric series implicit in (7) once one gets beyond their equivalent leading and next-to-leading terms. Both NSVZ and DRED schemes are presumably valid ones (although only the former upholds the Adler-Bardeen Theorem to all orders). One can even obtain a perturbative road-map between couplants in the two schemes: if $z \equiv x^{N S V Z}$ and $y \equiv x^{D R E D}$, then [4]

$$
y=z\left[1+\sigma z+\left(27+6 \sigma+\sigma^{2}\right) z^{2}+\left(351+117 \sigma+15 \sigma^{2}+\sigma^{3}\right) z^{3}+\ldots\right]
$$

where the constant $\sigma$ is arbitrary as a consequence of both (asymptotically-free) couplants having identical leading and next-to-leading terms within their respective $\beta$-function series.2] Consequently, unless one specifies initial values for couplants in each scheme, one cannot say anything at all about the relative size of couplants in the two different schemes at a given momentum scale. If, for example, the all-order extension of the DRED series (8) exhibits a pole, such a pole need not occur at its $x=1 / 6$ NSVZ location. Nevertheless, consistent infrared dynamics does require that a pole indeed occur in the DRED scheme. In other words, the all-orders extension of (8) should not exhibit the Landau-pole or IRFP behaviour seen to characterise of the $\beta$-function examples (1) and (5), respectively. Rather, the DRED scheme should the same infrared dynamics as are evident from (7): a finite infrared-attractive point $x\left(\mu_{c}\right)$ terminating the evolution of the asymptotically-free phase of the couplant, with $\mu_{c}$ serving as an infrared bound on the domain of $x(\mu)$.

\footnotetext{
${ }^{1}$ Equation (1) the $\beta$-function truncated after scheme-independent two-loop order terms, has already been used to generate an apparently self-consistent renormalization scheme [2].

${ }^{2} \mathrm{An}$ arbitrary constant in the relation between perturbative couplants in two different renormalization schemes of a given theory will occur provided the leading and next-to-leading terms of the $\beta$-function series in both schemes are the same. Such is the case, of course, for conventional QCD.
} 
To get some insight into the infrared behaviour of DRED supersymmetric gluodynamics, the four known terms of the $\beta$-function series (8) have been utilised to construct Padé approximants - ratios of polynomials in $x$ whose power-series expansions reproduce these known terms. Padé approximants are often employed not only to predict next order terms of a series, such as the $\beta$-function series for QCD [8], scalar field theory $[8,9,10]$, and supersymmetric QCD [4,11], but also to explore whether infinite series with only a few known terms can be expected to have zeros or poles [12]. [3 In reference [4], for example, it is demonstrated that every Padé approximant (except the truncated series itself) that reproduces the first four known terms of a series which differs infinitesimally from the geometric series $1 /(1-|r| x)$ exhibits a pole that

1) differs infinitesimally from the true pole at $x=1 /|r|$, and

2) occurs prior to any positive spurious Padé-approximant zeros.

In other words, the pole driven infrared dynamics of the NSVZ $\beta$-function (7) could have been predicted from Padé approximants constructed from that series' first four terms.

Relevant Padé approximants whose power series reproduce the four known terms of the DRED $\beta$-function (8) are $[4]$

$$
\begin{gathered}
\mu^{2} \frac{d x}{d \mu^{2}}=-9 x^{2} \frac{(1-14 x)}{\left(1-20 x+57 x^{2}\right)} \\
\mu^{2} \frac{d x}{d \mu^{2}}=-9 x^{2} \frac{\left(1-8.5714 x-24.4286 x^{2}\right)}{(1-14.5714 x)} \\
\mu^{2} \frac{d x}{d \mu^{2}}=-9 x^{2} \frac{1}{\left(1-6 x-27 x^{2}-810 x^{3}\right)}
\end{gathered}
$$

The first positive pole of (10) occurs at $x=0.0604$ and precedes the zero at $x=1 / 14$. Similarly, the pole of (11) at $x=1 / 14.5714=0.0686$ precedes that approximant's first positive zero $(x=0.0924)$, and the approximant $(12)$ exhibits a first positive pole at $x=0.0773$. Although (12) is constructed to have no zeros other than $x=0$, there is no a priori reason that this approximant should exhibit a positive pole of comparable magnitude to those of (10) and (11). These results (and further analysis of higher approximants presented in [4]) are clearly indicative of the same pole-driven infrared dynamics that characterise the NSVZ $\beta$-function (7) for the same $N=1$ supersymmetric SU(3) Yang-Mills field theory.

The techniques illustrated above are applicable to QCD itself. There is considerable controversy as to the infrared dynamics which characterise QCD, as well as the flavour dependence of such dynamics. The idea that the QCD couplant freezes out to some effective $\alpha_{s}(\mu=0)$ at sufficiently low momentum scales appears to have both some theoretical justification [14] (even for the $n_{f}=0$ case [15]) and as well as phenomenological utility [16]. However, such IRFP dynamics for $n_{f} \lesssim 6$ are inconsistent with both theoretical studies based upon a $\beta$-function series truncated after its first two scheme independent terms [17] as well as with a lattice study indicative of an $n_{f}=7$ threshold for IRFP dynamics [18].

A Padé-approximant approach to the infrared dynamics of QCD similar to the example presented above is formulated in detail in refs. [9], [13], and [19]. In this work, the known (and first unknown) terms of the QCD $\overline{M S}$ $\beta$-function series [20]

$$
\begin{aligned}
& \mu^{2} \frac{d x}{d \mu^{2}}=-\beta_{0} x^{2} S(x), \quad \beta_{0}=11 / 12-n_{f} / 6, \quad x \equiv \alpha_{s}(\mu) / \pi \\
S(x) & =1+\left[\left(51 / 8-19 n_{f} / 24\right) / \beta_{0}\right] x \\
& +\left[\left(2857 / 2-5033 n_{f} / 18+325 n_{f}^{2} / 54\right) / 64 \beta_{0}\right] x^{2} \\
& +\left[\left(114.23-27.134 n_{f}+1.5824 n_{f}^{2}+5.8567 \cdot 10^{-3} n_{f}^{3}\right) / \beta_{0}\right] x^{3} \\
& +R_{4} x^{4}+\ldots
\end{aligned}
$$

are used to construct various Padé-approximants whose leading positive zeros/poles are compared to see which occur first (or occur at all). We choose to use the $\overline{M S}$ renormalization shceme simply because the $\beta$-function series in this scheme is known to higher order than in any other perturbative scheme - moreover, phenomenological QCD is overwhelmingly based upon $\overline{M S}$ calculations. We denote by $S^{[N \mid M]}(x)$ the Padé-approximant to $S(x)$ whose

\footnotetext{
${ }^{3}$ Straightforward examples of this method for recovering the first positive pole or zero of $\sec (x) \pm \tan (x)$ are presented in ref. [13].
} 
numerator and denominator are respectively degree- $\mathrm{N}$ and degree-M polynomials of the couplant $x$. If $N+M=3$, the power-series expansion of the approximant $S^{[N \mid M]}(x)$ is of sufficiently high order to reproduce the known series terms in (14); e.g. for $n_{f}=3$

$$
\begin{aligned}
S^{[2 \mid 1]}(x) & =\frac{1-2.9169 x-3.8750 x^{2}}{1-4.6947 x} \\
S^{[1 \mid 2]}(x) & =\frac{1-8.1734 x}{1-9.9511 x+13.220 x^{2}}
\end{aligned}
$$

In both of these approximants, a positive pole precedes any positive zeros, indicative of the same pole-driven infrared dynamics known to characterise NSVZ supersymmetric gluodynamics. If one constructs such approximants for any choice of $n_{f}$ (as in ref. [13]), one finds for $n_{f} \leq 5$ that $S^{[2 \mid 1]}$ always has a positive pole preceding any positive zeros; moreover, the same statement applies to $S^{[1 \mid 2]}$ as well, provided $n_{f} \leq 6$. These results suggest that pole-driven infrared dynamics characterise QCD for up to five or six flavours. The occurrence of positive zeros that are not preceded by positive poles, (i.e., $\beta$-function zeros corresponding to IRFP dynamics) does not characterise $S^{[2 \mid 1]}$ until $n_{f} \geq 7$, and does not characterise $S^{[1 \mid 2]}$ until $n_{f} \geq 9$, corroborating the existence of a flavour threshold of IRFP behaviour $[17,18]$ as well as the absence of such behaviour below this threshold [21].

In ref. [13], these same qualitative conclusions are shown to be upheld by $S^{[N \mid M]}$ for $\mathrm{N}+\mathrm{M}=5$, i.e., for Padéapproximants to $S(x)$ whose power series are constructed to reproduce the four known terms of (14) and the arbitrary five loop term $R_{4} x^{4}$. In such approximants, each numerator and denominator polynomial coefficient of powers of $x$ is itself linear in the parameter $R_{4}-e . g$. for $n_{f}=3$

$$
S^{[2 \mid 2]}(x)=\frac{1+\left(7.1946-0.10261 R_{4}\right) x+\left(-11.329+0.075644 R_{4}\right) x^{2}}{1+\left(5.4168-0.10261 R_{4}\right) x+\left(-25.430+0.25806 R_{4}\right) x^{2}}
$$

Regardless of the value $R_{4}$ takes, one finds that positive zeros which precede every positive pole do not occur in the approximants $S^{[1 \mid 3]}, S^{[2 \mid 2]}$, or $S^{[3 \mid 1]}$ until $n_{f} \geq 9$, 7, and 6 , respectively, consistent with the non-occurrence of IRFP dynamics below these threshold values of $n_{f}$. Moreover, for arbitrary $R_{4}$ a positive pole which precedes every positive zero does occur in the approximants $S^{[1 \mid 3]}$ and $S^{[2 \mid 2]}$, provided $n_{f} \leq 5$. The approximant $S^{[3 \mid 1]}$ has a single positive pole only if $R_{4}>0$, a consequence of having a denominator linear in $x$, but this pole is found to precede any numerator zeros in the approximant for all positive values of $R_{4}$ as long as $n_{f} \leq 7$.

These results are clearly indicative of the occurrence of pole-driven infrared dynamics for QCD once heavy flavours are decoupled. Similar pole-driven infrared dynamics are also shown in [13] to characterise QCD in the $N_{c} \rightarrow \infty$ 't Hooft limit. Moreover, very recent work [18] has demonstrated that different approximants to the $N_{c}=3, n_{f}=3 \overline{M S}$ QCD $\beta$-function exhibit surprising consistency in their predictions of infrared boundary coordinates $\left(\mu_{c}, x\left(\mu_{c}\right)\right)$ associated with pole-driven infrared dynamics. The implications of such dynamics, in particular the possibility of having both a strong and an asymptotically-free phase of QCD with common infrared properties [6], have only begun to be explored. 


\section{Acknowledgements}

I am grateful to my research collaborators F. A. Chishtie, V. A. Miransky, and T. G. Steele, who coauthored of much of the research described above. I am also indebted to my deceased research collaborator Mark Samuel, who pioneered the application of Padé approximants to perturbative quantum field theory, and to Roger Migneron, whose final research paper [9] is the first published work in which Padé-approximants are applied to the infrared structure of QCD.

\section{References}

1. D. E. Groom et al. [Particle Data Group], Eur. Phys. J. C 15, 1 (2000).

2. G. 't Hooft, in Recent Developments in Gauge Theories, Vol. 59 of NATO Advanced Study Institute Series B: Physics, edited by G. t Hooft et al. (Plenum, N.Y., 1980).

3. D. R. T. Jones, Phys. Lett. B 123, 45 (1983).

4. V. Elias, J. Phys. G 27, 217 (2001).

5. V. Novikov, M. Shifman, A. Vainshtein, and V. Zakharov, Nucl. Phys. B 229, 381 (1983).

6. I. I. Kogan and M. Shifman, Phys. Rev. Lett. 75, 2085 (1995).

7. L. N. Avdeev, G. A. Chochia, and A. A. Vladimirov, Phys. Lett. B 105, 272 (1981); I. Jack, D. R. T. Jones, and A. Pickering, Phys. Lett. B 435, 61 (1998).

8. J. Ellis, M. Karliner, and M. A. Samuel, Phys. Lett. B 400, 176 (1997).

9. V. Elias, T. G. Steele, F. Chishtie, R. Migneron, and K. Sprague, Phys. Rev. D 58, 116007 (1998).

10. F. Chishtie, V. Elias, and T. G. Steele, Phys. Lett. B 466, 266 (1999); F. A. Chishtie and V. Elias, Phys. Lett. B 499, 270 (2001).

11. I. Jack, D. R. T. Jones, and M. A. Samuel, Phys. Lett. B 407, 143 (1997); J. Ellis, I. Jack, D. R. T. Jones, M. Karliner, and M. A. Samuel, Phys. Rev. D 57, 2665 (1998).

12. G. Baker and P. Graves-Morris, Pad Approximants [Vol. 13 of Encyclopedia of Mathematics and its Applications] (Addison-Wesley, Reading, MA, 1981) pp. 48-57.

13. F. A. Chishtie, V. Elias, V. A. Miransky, and T. G. Steele, Prog. Theor. Phys. 104, 603 (2000).

14. A. C. Mattingly and P. M. Stevenson, Phys. Rev. Lett. 69, 1320 (1992); P. M. Stevenson, Phys. Lett. B 331, 187 (1994).

15. A. C. Mattingly and P. M. Stevenson, Phys. Rev. D 49, 437 (1994).

16. Yu. L. Dokshitzer, in Proceedings of the 29th International Conference in High Energy Physics, A. Astbury, D. Axen, and J. Robinson, eds. (World Scientific, Singapore, 1999) pp. 305-324.

17. T. Banks and A. Zaks, Nucl. Phys. B 196, 189 (1982); T. Appelquist, J. Terning, and L. C. R. Wijewardhana, Phys. Rev. Lett. 77, 1214 (1996); V. A. Miransky and K. Yamawaki, Phys. Rev. D55, 5051 and (Err.) D 56, 3768 (1997).

18. Y. Iwasaki, K. Kanaya, S. Sakai, and T. Yoshi, Phys. Rev. Lett. 69, 21 (1992).

19. F. A. Chishtie, V. Elias, and T. G. Steele, Phys. Lett. B 514, 279 (2001).

20. T. van Ritbergen, J. A. M. Vermaseren, and S. A. Larin, Phys. Lett. B 405, 323 (1997).

21. E. Gardi, G. Grunberg, and M. Karliner, JHEP 9807, 007 (1998). 